\section{Schizophrenia-like psychosis in African and Caribbean elders}

The interesting study reported by Reeves $e t$ al (2001) draws attention to mental health service provision for ethnic minority elders. However, their findings could be misleading as they repeat common errors of cross-cultural research.

By definition, African and Caribbean elders are not a homogeneous group. Neither are they synonymous with 'AfricanCaribbean'. As a population, they are of different history, ethnicity and culture. Furthermore, as migrants from different geographical regions of the world it is important that their different identities are appreciated, especially in their 'third age'. Unlike the melting pot of second and third generations, these elders maintain distinct values that influence their social and helpseeking interactions. Migration pathways between the groups are diverse as well, ranging from long-term to recent, academic pursuit to meeting labour needs and the 'culture-shocked' to the assimilated.

There are also fundamental problems with defining cases by place of birth. 'African-born' includes Algerians, Egyptians, East African Asians and White South Africans. Similarly, 'Caribbean-born' persons of African, Asian and mixed-race provide a richly heterogeneous population of elders. How does one draw meaningful scientific conclusions?

The authors did not clarify the proportion of subjects from each of the African and Caribbean groups in the study. This may influence analysis and outcomes. One must also make a clear distinction between onset of illness and contact with services. First contact above age 65 years does not necessarily imply late onset of illness as alternative care pathways and help-seeking patterns may prevail (e.g. years of Pentecostal church attendance). Psychotic symptoms may go undisclosed for many years, particularly among groups suspicious or mistrusting of mental health services. On the other hand, reports of witchcraft or communication with ancestors, previously culturally sanctioned, may be mistaken for psychotic (or schizophrenia-like) experiences.

The authors argue that referral bias by primary care and community physicians is unlikely, as evidenced by low contact rates for anxiety disorders and depression. This is of concern, however, as other researchers (Abas, 1996; Shah, 1998) have reported underdiagnosis of these disorders in ethnic minority populations, with a focus on psychotic and behavioural over affective symptoms.

Although Reeves et al opine that rates may be influenced by social isolation, physical ill health and social exclusion, this was not supported by evidence from their study.

The conclusions of this study are by no means generalisable and highlight ethnic and cultural confusion, as well as the neglect of depression and anxiety disorders, in research involving African and Caribbean elders. In the words of an elderly African, 'if the heart is too heavy with sorrow, it may disturb the mind'. As clinicians we must not ignore this cry.

Abas, M. (1996) Depression and anxiety among older Caribbean people in the UK: screening unmet need and the provision of appropriate services. International Journal of Geriatric Psychiatry, II, 377-382.

Reeves, S. J., Sauer, J., Stewart, R., et al (200I) Increased first-contact rates for very-late-onset schizophrenia-like psychosis in African- and Caribbeanborn elders. British Journal of Psychiatry, 179, 172-174.

Shah, A. K. (1998) The psychiatric needs of ethnic minority elders in the United Kingdom. Age and Ageing 27. 267-269.

O. A. Ayonrinde Maudsley Hospital, Denmark Hill, London SE5 8AZ, UK

Authors' reply: We thank Dr Ayonrinde for raising the issue of heterogeneity within ethnic groups and would fully agree that this is particularly pertinent to the comparison groups chosen for our study. Very-late-onset schizophrenia-like psychosis is a relatively rare disorder and large populations would have to be surveyed to make any reasonable estimate of community incidence rates. The method we employed involved the enumeration of referrals to secondary care over a defined period and the estimation of source populations using census-derived data. In our casenote study, the ethnicity of referrals was defined by objective criteria (birthplace). However, subjective criteria were used in the 1991 census coding. For example, a person born in the Caribbean might choose to define him- or herself in their census return as Black Caribbean, Black African or Black Other. For this reason, we decided to include all African- and Caribbean-born referrals and all Black groups within the denominator populations. In fact, $96 \%$ of referrals were Caribbean-born and there are relatively few African-born people within the age ranges considered in south London.

All populations are heterogeneous, whether these are defined according to geography, culture, religion or shared ancestry. For research involving ethnic groups, the purist might argue that the only solution would be not to attempt any comparisons between categories, which will always be inadequate. This would mean that the evidence base for service provision and public health interventions would be derived entirely from the majority population. A fundamental objective of epidemiology is to describe and explain the distribution of health states across populations. Our findings suggest that incidence rates for an important disabling disorder are markedly different between two coarsely defined populations. Further research is clearly required to refine and explain this observation.

We cannot exclude from our data the possibility that a specific referral bias existed in relation to psychotic symptoms in African- and Caribbean-born individuals. However, for this alone to have accounted for the observed differences, the bias would need to have been at least ten times greater for African and Caribbean individuals. This would not accord with our experience of referral patterns to an old age psychiatry service in south London.

S. Reeves, R. Stewart, R. Howard Institute of Psychiatry, De Crespigny Park, Denmark Hill, London SE5 AF, UK

\section{Attitudes to depot antipsychotics}

Walburn et al (2001) are correct to place their conclusions regarding patients' favourable attitude to depot antipsychotic medication in the context of the paucity of studies using unselected patient samples. Indeed, depot preparations tend often to be used for patients who are otherwise unlikely and/or unwilling to accept antipsychotic medication, and a true reflection of patient attitudes to depot antipsychotics can be established only from research based on representative samples of patients with psychotic illnesses.

In Australia, the study of Low Prevalence (Psychotic) Disorders (Jablensky et al, 2000), conducted in 1997-1998, allowed an arguably more accurate evaluation of patients' attitudes to depot medication. In this study, 998 persons with a non-organic psychosis were randomly selected from all 